\title{
Mobile Backhaul in Release 8 and Beyond: Benefits, Challenges, Market Status and Impact Analysis
}

\author{
Tadele Abera1*, Firew Dereje2, Biruk Larago1 \\ ${ }^{1}$ Dire Dawa University, DDIT, Dire Dawa, Ethiopia \\ ${ }^{2}$ Mettu University, Mettu, Ethiopia \\ E-mail address: tadenegn@gmail.com
}

Received: 05 November 2020; Accepted: 25 November 2020; Published: 08 December 2020

\begin{abstract}
The significantly increasing mobile capacity demand, the use of advanced techniques like enhanced multiple input multiple output to boost throughput, very high data rate, reliability, very low latency, long battery life, mobility, and to enable internet of things in $4 \mathrm{G}$ and $5 \mathrm{G}$ technologies, aggravate extreme backhaul requirements with respect to capacity, latency, availability, energy, and on the top of this, cost efficiency. However, it is challenging to get a complete one backhaul technology that can solve all problems of backhaul especially massive introduction of small cells in mind. To overcome various backhaul problems there are many backhaul solutions i.e. the use of diversified backhaul under different use cases provides cost efficient backhaul solution. This paper presents benefits, implementation challenges, and use cases of mobile backhaul in release 8 and beyond. It also presents coverage and capacity analysis using excel based dimensioning tool for a given sample area. Lastly the paper shows economic benefits of diversified backhaul for small and macro cells and high-level implementation strategy for the considered area.
\end{abstract}

Index Terms: 5G, LTE, LTE-A, Millimetre wave, Mobile backhaul, Small cells, Sub-6 GHz, TVWS.

\section{Introduction}

The backhaul network in cellular networks can be defined as a transport network that connects base stations to controllers and mobile core network elements. It can also connects the eNBs to other eNBs as far as $4 \mathrm{G}$ and beyond technologies are concerned. Mobile backhaul technologies consists currently available dedicated fibre, copper, licensed sub-6 GHz (bellow $6 \mathrm{GHz}$ ), unlicensed sub-6 GHz $(2.4 \mathrm{GHz}, 5.8 \mathrm{GHz})$, microwave $(6-42 \mathrm{GHz})$, and satellite links $[1,2,3,4,5]$. In addition, it consists of newly emerging wireless technologies like licensed millimetre wave (70-80 GHz), unlicensed millimetre wave $(60 \mathrm{GHz})$ and TV white space $(470-694 \mathrm{MHz})[4,5]$.

Release 8 and beyond technologies improvement in data rate, capable of supporting multi-traffic volumes, use of advanced techniques like enhanced multiple input multiple output (MIMO) to boost throughput, very high spectral efficiency, reliability, reasonably very low latency, long battery life, mobility, and suitability to enable internet of things (IOT) are factors that play an important role in shifting backhaul architectures from earlier versions like asynchronous transfer mode (ATM), time division multiplexing (TDM) to an all IP model.

IP based backhaul that replaced earlier versions in long term evolution (LTE), long term evolution-advanced (LTE-A) and 5G makes the network management easier and provides a better environment to incorporate IP technologies for routing and quality of experience. With a lot of advancements in LTE, LTE-A and 5G particularly associated with small cells in mind: backhaul is a driving force to use and go further on sub-6GHz, millimeter wave band, TV white space (TVWS) bands, even widely used microwave. Because of very little congestion, millimeter wave bands are possible solution for small cell backhaul. They are very compatible with a short small cell ranges, due to their high frequencies. Similarly, higher microwave frequencies also draw attention to use for small cells backhaul $[1,2,3,4,5]$.

Even though backhaul associated with release 8 and beyond has considerable benefits, there are some implementation challenges. Some of them have been addressed as follows: Scalability: to support massive number of base stations at higher capacities the backhaul network must be smoothly scaled $[4,5,6]$. This is one implementation challenge. Flexibility: the introduction of small cells in addition to macro cells in an LTE, LTE-A and 5G environment brings the need to leverage cost-efficient backhaul access infrastructure that can meet required quality of experience. Due to this backhaul access types will be greatly diversified. This in turn drives a requirement for more flexible backhaul solutions. In addition to LTE, LTE-A and 5G, it should support for $2 \mathrm{G}$ and $3 \mathrm{G}$ services when needed $[4,5,6]$. 
Simplicity: to reduce the total cost of ownership (TCO) operations should be simplified. Moreover, it should permit efficient network deployment, administration and maintenance.

This paper addresses: which backhaul technology demand is increasing and which one demand is decreasing i.e. which backhaul technology is intensively or rarely used for small or macro cellular network. It also addresses when and where different backhaul solutions can be used. Coverage and capacity analysis by counting small cells and macro cells for diversified backhaul has been also addressed. Economic benefits using diversified backhaul for small cells and macro cells has been shown. Since there is no one complete backhaul solution that can overcome all backhaul problems, this paper proposed to take the full advantage of diversified backhaul.

The rest of the paper is organized as follows. Section II presents market share of backhaul networks. Section III presents impact analysis of backhaul technology. Section IV, high level implementation strategy, and section V concludes the paper.

\section{Market Share}

It is well known that market share is an important parameter when measuring the successfulness of backhaul business, and hence it has been introduced as one section of this paper. [1,2,3,4,5,6,7,8,11,12,13,14] shows the usage of macro and small cell backhaul technologies over the couple of years. From the research papers it can be seen that millimeter wave is going to be intensively used for both small and macro cell backhaul worldwide. Also, the demand of fiber for both small cell and macro is increasing due to its unlimited capacity. The macrocell microwave LTE backhaul market will not grow at the same rate as small cell microwave backhaul as it shows consistent growth. Licensed sub-6 $\mathrm{GHz}$ for non-light of sight (NLoS) is proved viable small cell backhaul solution [5] [7]. Satellite backhaul technology will remain possible solution, considering some deployments in rural areas where there is no line of sight to macro or other cable infrastructure.

\section{Mobile Backhaul Impact Analysis}

In this section relevance of mobile backhaul; use cases; and coverage and capacity analysis has been discussed.

\subsection{Relevance of mobile backhaul}

The significantly increasing data volumes and highly innovative devices will drive the continued need for additional backhaul for mobile operators [8,9]. To accommodate both access and core network traffic, backhaul network capacity need to be improved. To this end appropriate backhaul technology should be applied in different use cases. The existing usage and deployment strategy of backhaul will not satisfy the desired network requirement of release 8 and beyond [10]. If we use traditional microwave as the only solution it will not provide sufficient capacity because of very narrow channel space. The same will happen if we use sub-6 GHz [8]. The cost of fiber including installation is high even though it has a large bandwidth. The situation is further exacerbated with massive introduction of small cells as the backhaul network is bottleneck for its deployment. These factors are leading mobile operators to use diversified backhaul (as shown in Fig. 1) technologies over conventional backhaul. Thus, diversified backhaul will meet transport capacity requirement as various mobile operators are in a way of commercially launching $5 \mathrm{G}$ in 2020 .

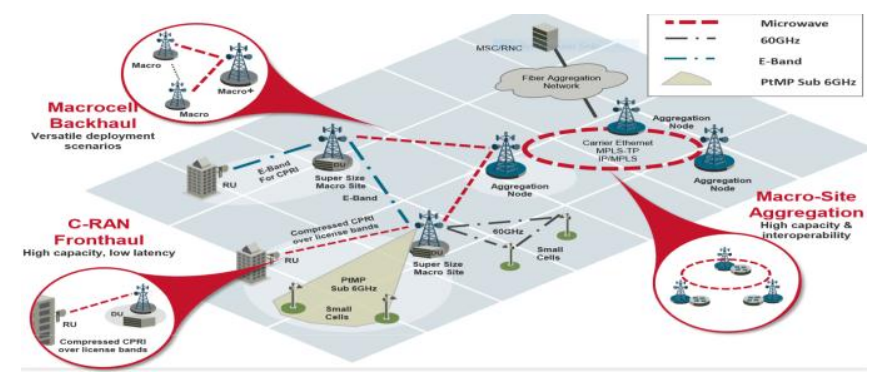

Fig. 1. Diversified backhaul [9]

\subsection{Use cases}

Use cases can be termed in this document to show where backhaul technologies can be used. Millimetre wave $(60 \mathrm{GHz})$ is used to backhaul small cells to street level i.e. street to street or street to rooftop links. Millimetre wave (70$80 \mathrm{GHz}$ ) is used to aggregate small cells at the rooftop under microcellular sites. Microwave bands is deployed at the point where line of sight to hub is available. Sub- $6 \mathrm{GHz}$ (licensed) is deployed to enable the rapid deployment of large areas of small cells. Sub-6 GHz (unlicensed) can be deployed for remote or isolated locations. TVWS can be used as backhaul infrastructure between small cells in locations where TVWS channel availability is high. Satellite can be used for targeted capacity hotspots for locations where there no fiber infrastructure or line of sight to macro. Fiber to cabinet 
or exchange (FTTX) can be suitable solution for Hotspot, indoor coverage, outdoor coverage where available $[6,7,8,11,12,13]$.

\subsection{Coverage and capacity analysis}

As a sample an area of $23000 \mathrm{~m}^{2}$ was taken with total population at peak time approximately 900 . This area to be covered with small cells and one microcell using diversified backhaul. For this analysis, excel based dimensioning tool from ALCatel Training LTE with original title: 271545062 -LTE-Rel8-Downlink-RLB-2014-4x4 has been used. From the tool allowed propagation loss and downlink cell range can be obtained and used for coverage and capacity analysis.

\begin{tabular}{l|r|}
\hline DL Link budget for LTE & \\
\hline & \\
\hline eNodeB - UE & \\
eNB Transmitter characteristics & 2 \\
Number of PRBs & $2.00 \mathrm{Mbits} / \mathrm{s}$ \\
Downlink data rate & $46.00 \mathrm{dBm}$ \\
eNodeB TX power & $8.00 \mathrm{dBi}$ \\
eNodeB antenna gain & $2.00 \mathrm{~dB}$ \\
eNodeB antenna cable loss & $\mathbf{5 2 . 0 0 \mathrm { dBm }}$ \\
EIRP & \\
& \\
\hline UE receiver characteristics & $7.00 \mathrm{~dB}$ \\
UE noise figure & $-104.43 \mathrm{~dB}$ \\
Thermal noise & $-97.43 \mathrm{dBm}$ \\
Receiver noise floor & $42.22 \mathrm{~dB}$ \\
Required SINR & $-55.21 \mathrm{dBm}$ \\
Receiver sensitivity & $1.00 \mathrm{~dB}$ \\
Control channel overhead & $1.00 \mathrm{~dB}$ \\
Rx antenna gain & $0.00 \mathrm{~dB}$ \\
Body loss & $7.00 \mathrm{~dB}$ \\
Shadowing loss & $4.00 \mathrm{~dB}$ \\
Interference margin & $10.00 \mathrm{~dB}$ \\
Indoor penetration loss & $\mathbf{8 6 . 2 1 ~ d B}$ \\
Allowed propagation loss & $1.4 \mathrm{E}-23$ \\
\hline Boltzmann constant &
\end{tabular}

Fig. 2. Allowed propagation loss

\begin{tabular}{lc}
\hline Range (Okumura-Hata path loss model) & \\
\hline Carrier frequency & $2600 \mathrm{MHz}$ \\
BS antenna height & $10 \mathrm{~m}$ \\
MS antenna height & $2.5 \mathrm{~m}$ \\
MS antenna gain function (large city) & 1.92587 \\
MS antenna gain function (small city) & 3.11382 \\
MS antenna gain function (suburban) & 16.2584 \\
MS antenna gain function (rural) & 37.2644 \\
Path loss exponent & 38.35 \\
Path loss constant (large city) & $146.322 \mathrm{~dB}$ \\
Path loss constant (small city) & $145.134 \mathrm{~dB}$ \\
Path loss constant (suburban area) & $131.989 \mathrm{~dB}$ \\
Path loss constant (rural area) & $110.983 \mathrm{~dB}$ \\
Path loss constant (rural Saarijarvi) & $120.983 \mathrm{~dB}$ \\
Downlink range & \\
Urban city & $0.03 \mathbf{~ k m}$ \\
Small city & $0.03 \mathbf{~ k m}$ \\
Suburban & $0.06 \mathbf{~ k m}$ \\
Rural & $0.23 \mathbf{~ k m}$
\end{tabular}

Fig. 3. Downlink cell range

For coverage analysis, the range (radius) is $30 \mathrm{~m}$ from Okumura-Hata path loss model. Area of a single cell can be calculated using (1):

$$
A=\frac{3}{2} N R^{2} \sqrt{3}
$$

where:

$A=$ Area

$R=$ Radius of the cell

$N=$ Cluster size

$\mathrm{R}=30 \mathrm{~m}$ 
Area of single cell $(1)=2338.2 \mathrm{~m}^{2}$ and Total area $=23000 \mathrm{~m}^{2}$. Then the required number of base stations to cover area can be total area divided by single cell area which is 10 .

For capacity analysis, assuming $90 \%$ of the total population is covered: $0.9 * 900=810$. Assuming Overbooking factor of 50. Then simultaneously supported users to be

$$
810 / 50=17
$$

Assuming 3 small cell users supported by one small cell

$$
\frac{17}{3}=6
$$

The number of base stations for capacity is 6 and which is less than base stations for coverage. This shows capacity can be effectively handled.

\section{Economic Benefits}

It has been shown using the following simple layout how network to be backhauled to show economic benefits of given area. Two scenarios have been considered to show economic benefits of diversified backhaul in the sample area. The first: assuming macro cell to aggregation point 2 is fiber and remaining links are point to point (PtP) microwave.

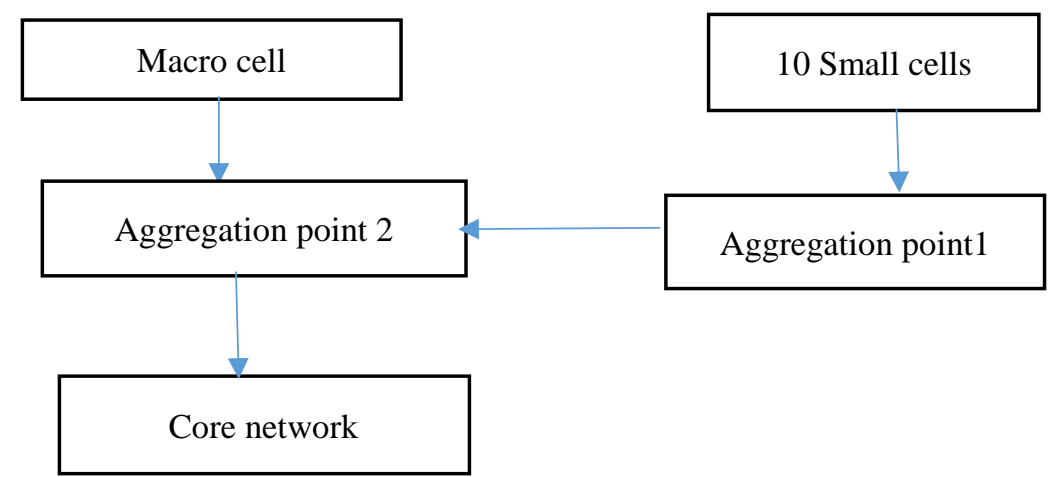

Fig. 4. Sample backhaul network layout

The second: assuming macro cell and aggregation point 2 backhauled by licensed millimetre wave band PTP and aggregation point 2 and core network backhauled by PTP microwave and rest is backhauled by point to multipoint (PTMP) microwave.

Table 1. Scenario 1

\begin{tabular}{|ll|}
\hline Capex of fiber & Cost (USD) \\
$\begin{array}{l}\text { Equipment cost: connection setup } \\
\text { Network planning, site acquisition, } \\
\text { installation }\end{array}$ & 2000 \\
& 2000 \\
& (RAN site) \\
Opex of fiber & \\
leased line fees (per year) & 140,000 \\
Other recurring operating costs & 1000 \\
(power, maintenance, etc.) & (RAN site) \\
$\begin{array}{l}\text { Total cost of fiber at 5 years }=2000+2000+140,000 * 5 \\
\text { +1000 = USD 705000 }\end{array}$ & \\
Capex of PTP MW & \\
$\begin{array}{l}\text { Equipment costs, outdoor per link } \\
\text { Equipment costs, indoor }\end{array}$ & 11500 \\
$\begin{array}{l}\text { Network planning, site acquisition, } \\
\text { installation per link }\end{array}$ & 9000 \\
$\begin{array}{l}\text { Opex of PTP MW } \\
\text { Site rental fees (per year): Outdoor } \\
\text { equipment and indoor equipment } \\
\text { respectively }\end{array}$ & 8700 \\
Spectrum licensing fees & 7000 per link, \\
& 1500 per link \\
$\begin{array}{l}\text { Other recurring operating costs } \\
\text { (power, maintenance, etc.) }\end{array}$ & 5418 per link (14 \\
\end{tabular}


Total cost for PTP MW at 5 years $=11500 * 10+9000+8700+7000 * 1 * 5+1500 * 10 * 5+5418 * 11+8000 * 11=390298$ USD. Then total cost of fiber + PTP MW at 5years $=390298+705000=1095298$ USD

Table 2. Scenario 2

\begin{tabular}{|ll|}
\hline Capex of licensed millimeter wave & Cost (USD) \\
band PTP & \\
Equipment costs, outdoor per link & 19500 \\
Equipment costs, indoor & 9000 \\
$\begin{array}{l}\text { Network planning, site acquisition, } \\
\text { installation per link }\end{array}$ & 8700 \\
Opex of licensed millimetre wave band PTM \\
Site rental fees (per year): Outdoor \\
equipment and indoor equipment \\
respectively
\end{tabular}

AS it can be seen from the total cost, the first scenario costs 1.09 million USD and second costs 0.44 million USD. This reveals that if we diversify backhaul technologies more and select appropriate one will lead cost efficient backhaul network.

\section{High Level Implementation Strategy}

High level implementation strategy of backhaul network for given area can be shown in tabular form as follows 
Table 3. High level implementation strategy

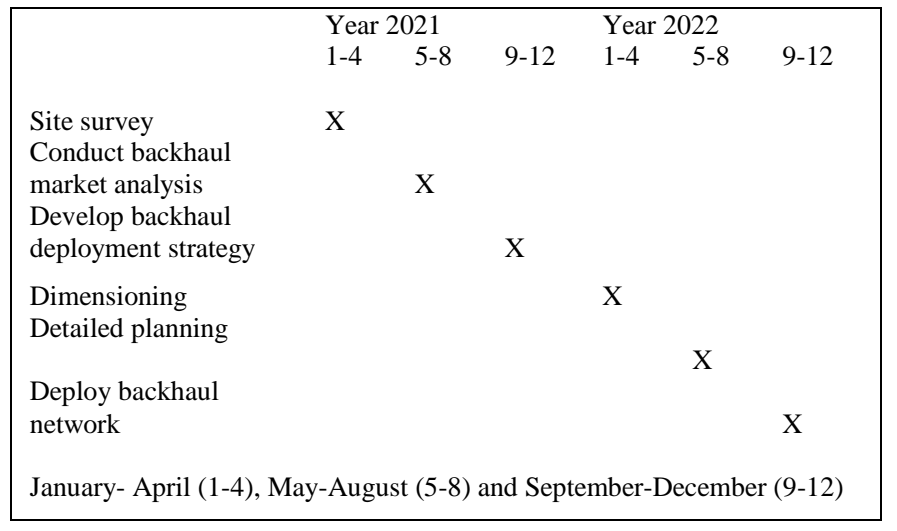

\section{Conclusion}

It is very important to upgrade the performance of backhaul network as $4 \mathrm{G}$ and $5 \mathrm{G}$ technologies requires very low latency, very high data rate, and reliability. All IP backhaul simplifies management, results cost savings and easier maintenance. Challenges associated with backhaul enhanced the stake holders to go further on emerging wireless technologies to get additional backhaul. The market status of emerging wireless technologies like millimetre wave shows promising future solution for backhaul as it has been shown in aforementioned review materials. Since there is no one complete backhaul solution that can overcome all backhaul problems, diversified backhaul in this research has been proposed as cost efficient solution that helps to meet high transport requirement capacity. To this end, it has been estimated based on OFCOM and Senza report that backhaul capex and opex for two scenarios for given site to show cost efficiency of diversified backhaul. The cost estimation shows that more diversified and appropriate backhaul technology will give most cost-efficient solution.

\section{References}

[1] Mona Jaber et al., "5G backhaul challenges and emerging research directions: A survey," IEEE Access, 2016.

[2] Uzma Siddique et al, "Wireless backhauling of 5G small cells: challenges and solution approaches," IEEE wireless commun., 2015.

[3] Renaud Alexandre et al, "Full duplex self-backhauling for small cells 5G networks," IEEE wireless commun., 2015.

[4] Ming Xiao et al, "Millimeter wave communications for future mobile networks," IEEE J. Sel. Areas Commun., vol. $35,2017$.

[5] GSMA, "Wireless backhaul spectrum policy, recommendations and analysis," white paper, 2014.

[6] Alcatel-lucent," Backhaul considerations for LTE and LTE-A," white paper, 2013.

[7] Ofcom, "Business connectivity market review," white paper, 2015.

[8] Analysys mason, " Mobile backhaul market: Phase 1 report," Report for Vodafone, 27 February 2014.

[9] Imran Khan, 'Efficient low-overhead channel estimation for 5G lens based millimetre-wave massive MIMO systems,' I.J. Wireless and Microwave Techniques, 2018.

[10] B.Lalitha, 'Efficient techniques to reduce effects of topology mismatch and heterogeneity in unstructured P2P networks,' I.J. Wireless and Microwave Techniques, 2016.

[11] Ceragon, "Resolve hauling challenges for our customers," Jefferies 2013 Global Technol. Media and Telecom Conf., May 2013.

[12] Small cell forum, "Backhauling technologies for small cells: use cases, requirements and solutions," white paper, February 2013.

[13] Senza "Crucial economics for mobile data backhaul," white paper, 2013.

[14] S.K.Agrawal, Kapil Sharma," Intelligent software defined atmospheric effect processing for $5^{\text {th }}$ generation (5G) millimetre wave (MM Wave) communication system,"' I.J. Wireless and Microwave Techniques, 2018.

\section{Authors' Profiles}

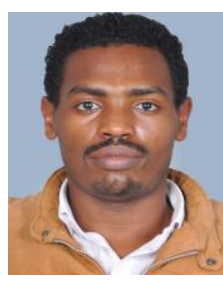

Tadele Abera has received BSC degrees from Hawassa University, Ethiopia by Electrical Engineering in 2011 and MSC degrees from Addis Ababa University, Ethiopia by Electrical Engineering (Communication Engineering Specialization) in 2014. He was test and commissioning Engineer in different electric power upgrading projects in Ethiopia. He is currently lecturer in Dire Dawa University, Ethiopia, under School of Electrical and Computer Engineering. He has publications on ICAST 2019. His research interests are cognitive radio, massive MIMO, millimetre wave communication, backhaul networks and interference management techniques. His research interest extends to power systems and control including power reliability, power quality, PID controllers and MPC. 


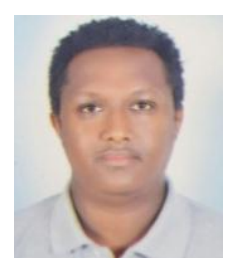

Firew Dereje has received BSC degrees from Hawassa University, Ethiopia by Electrical Engineering in 2013 and MSC degrees from Addis Ababa Science and Technology University, Ethiopia by Electrical Engineering (Control Engineering Specialization) in 2019. He is currently lecturer and head of electrical and computer engineering in Mettu University, Ethiopia, under Faculty of Engineering and Technology. He has publication on ICAST 2019. His research interests are backhaul networks, power reliability, power quality, PID controllers and MPC.

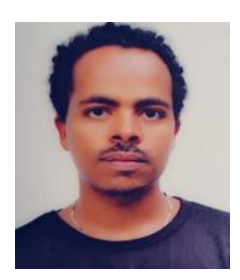

Biruk Larago has received MSC degrees from Adama Science and Technology University, Ethiopia by Electrical Engineering (Power Electronics Specialization) in 2018. He is currently lecturer and head of Power Engineering in Dire Dawa University, Ethiopia, under School of Electrical and computer Engineering. His research interests are backhaul networks, power reliability, power quality, PID controllers, MPC and FACTS.

How to cite this paper: Tadele Abera, Firew Dereje, Biruk Larago, " Mobile Backhaul in Release 8 and Beyond: Benefits, Challenges, Market Status and Impact Analysis", International Journal of Wireless and Microwave Technologies(IJWMT), Vol.10, No.6, pp. 9-15, 2020.DOI: 10.5815/ijwmt.2020.06.02 